\title{
Results from Mice Step IV
}

\section{François Drielsma*}

MICE Collaboration

E-mail: francois.drielsma@unige.ch

Muon beams of low emittance provide the basis for the intense, well characterised neutrino beams of the Neutrino Factory and for lepton-antilepton collisions at energies of up to several $\mathrm{TeV}$ at a Muon Collider. The international Muon Ionization Cooling Experiment (MICE) will demonstrate ionization cooling, the technique by which it is proposed to reduce the phase space volume occupied by the muon beam.

MICE is being constructed in a series of Steps. The configuration currently in operation at the Rutherford Appleton Laboratory is optimised to study the properties of liquid hydrogen and lithium hydride that affect cooling. The data taken in the present configuration have been partially analysed and the available results will be described in detail.

The European Physical Society Conference on High Energy Physics

5-12 July

Venice, Italy

${ }^{*}$ Speaker. 


\section{Introduction}

Intense muon sources are required for a future Neutrino Factory or Muon Collider [1, 2]. Conventional cooling techniques applied to muon beams [3] would leave almost no muons to be accelerated since the muon lifetime is short $\left(\tau_{\mu} \sim 2.2 \mu \mathrm{s}\right.$ ). Ionization cooling [4] is the only technique that operates quickly enough to achieve the necessary phase space contraction with manageable losses. A factor of close to a million in 6D muon cooling has been reached in a simulation [5]. The rate of change in normalised emittance when passing through an absorber can be approximated by the following "cooling equation" [4]

$$
\frac{d \varepsilon_{N}}{d s} \simeq-\frac{\varepsilon_{N}}{\beta^{2} E_{\mu}}\left|\frac{d E_{\mu}}{d s}\right|+\frac{\beta_{\perp}(13.6 \mathrm{MeV})^{2}}{2 \beta^{3} E_{\mu} m_{\mu} c^{2} X_{0}},
$$

where $\beta_{\perp}$ is the transverse optical Twiss function, $\beta c, E_{\mu}, m_{\mu}$ are the particle velocity, energy and mass, and $X_{0}$ is the absorber radiation length.
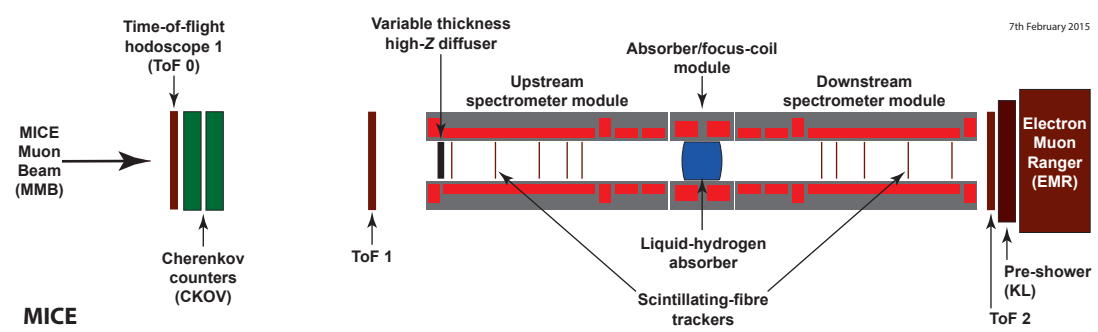

Figure 1: Schematics of the MICE experiment at present.

The Muon Ionization Cooling Experiment [6] is studying the cooling equation in detail and for a variety of input beams, magnetic lattices and absorbers to demonstrate the feasibility of this technique. A schematic of the experiment in its Step IV configuration is shown in figure 1. The particles produced in the MICE beam line first pass through the upstream particle identification (PID) apparatus, are sampled upstream and downstream of the absorber by scintillating fibre trackers before being dumped in the downstream PID section [7, 8, 9, 10].

MICE is a single-particle experiment. The phase space evolution is reconstructed by measuring the vector $\left(x, y, p_{x}, p_{y}, p_{z}\right)$ of each selected muon before and after going through the absorber. Tracks are accumulated into a beam ensemble during the analysis process. Figure 2 shows a two dimensional projection of 25 simulated muon trajectories going through the MICE experiment.

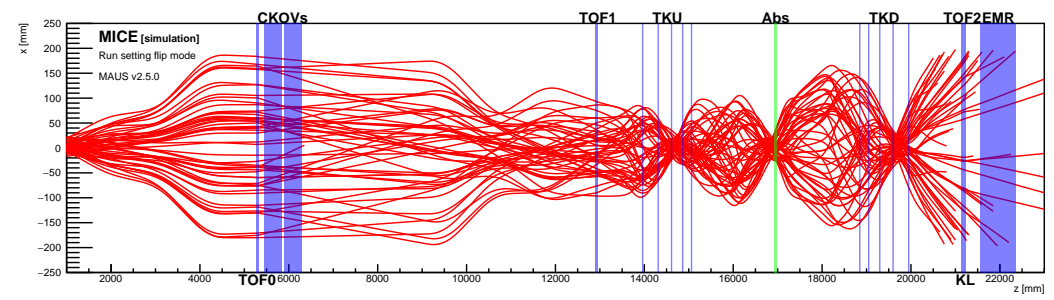

Figure 2: Sample of 25 simulated muon trajectories in the MICE experiment. A tight focus, i.e. low $\beta_{\perp}$, can be observed at the level of the absorber (green) to minimize the heating term of the cooling equation. 


\section{Direct measurement of the MICE Muon Beam emittance}

The MICE Muon Beamline delivers a high purity beam with less than $1.4 \%$ pion contamination [9]. The flight time between the first two time-of-flight hodoscopes (TOF0 and TOF1) is used to remove other contamination sources such as decay positrons from the final sample. Track pattern recognition and a Kalman filter provide the best estimate of the particle position and momentum in the station closest to the absorber [11] (reference plane).

The time-of-flight between TOF0 and TOF1 is represented as a function of the total momentum reconstructed in the tracker in figure 3. The dotted line represents the ideal time-of-flight of a muon that underwent the Bethe-Bloch most probable momentum loss between entering TOF1 and reaching the tracker reference plane.

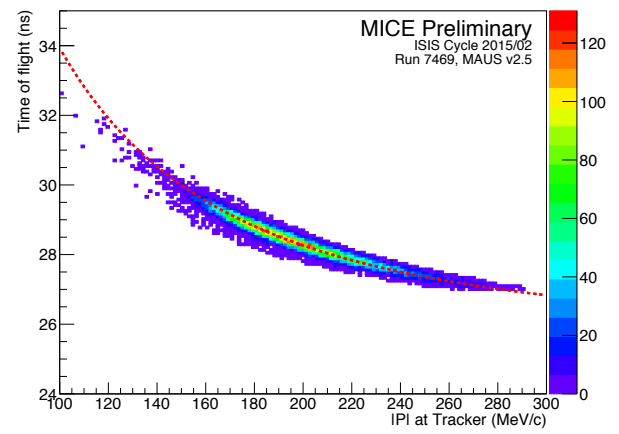

Figure 3: Time-of-flight distribution between TOF0 and TOF1 as a function of the total momentum measured in the upstream tracker.

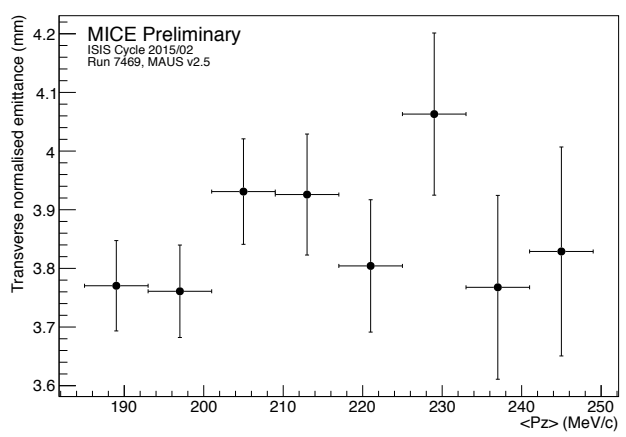

Figure 4: Transverse normalised emittance of $8 \mathrm{MeV} / c$ longitudinal momentum bins.

Due to the large aperture dipoles used to select a momentum range of the muon distribution, the transported sample of muons contains significant dispersion in the horizontal plane. The sample shows a large momentum spread, $\sigma_{p_{z}} \simeq 25.7 \mathrm{MeV} / c$, for a mean longitudinal momentum, $\left\langle p_{z}\right\rangle=$ 195.4 MeV/c. The emittance of the whole particle sample does not accurately represent the volume occupied by the particles in the phase space, as muons with different longitudinal momenta occupy different regions of the transverse phase space. To remove the effect of the dispersion, the particle sample was divided into momentum-coherent $8 \mathrm{MeV} / c$ bins.

The transverse normalised emittance was calculated for each $8 \mathrm{MeV} / c$ bin as

$$
\varepsilon_{N}=\frac{1}{m_{\mu}} \sqrt[4]{\operatorname{det} \Sigma}
$$

with $m_{\mu}$ the muon mass and $\Sigma$ the 4D transverse phase space covariance matrix.

The measured transverse normalised emittance is represented for each momentum bin in figure 4. The horizontal error bars represent the limits of each momentum slice while the vertical error bars are purely statistical errors on the measurements. Systematic error studies are under way but preliminary results indicate that they are small in comparison. The binned emittances are consistent across the range of studied momenta. The mean measured transverse normalised emittance for the whole sample is $3.85 \pm 0.04 \mathrm{~mm}$. 


\section{Measurement of the scattering distributions}

Results from MuScat [12] indicate that the effect in low Z materials is not well modelled in simulations such as GEANT4 [13]. MICE will therefore measure the multiple Coulomb scattering distribution to validate the scattering model and understand the heating term in equation 1.1, in order to make more realistic predictions of the emittance reduction.

MICE has collected straight track data for muon beams at three different momenta, $172 \mathrm{MeV} / \mathrm{c}$ (in order to compare with MuScat), $200 \mathrm{MeV} / c$ and $240 \mathrm{MeV} / c$ with and without the lithium hydride $(\mathrm{LiH})$ absorber in place (thickness of $65 \mathrm{~mm}, X_{0}=79.62 \mathrm{~g} \mathrm{~cm}^{2}$ ). The position and momentum of each muon is measured by the trackers and the time-of-flight, the latter also provides particle identification. Selection criteria on the tracks were imposed to select a pure, well understood sample. Bayesian deconvolution was applied to the selected data in order to extract the scattering distribution within the absorber material and comparisons have been made to GEANT4 as well as to a standalone scattering model developed by Carlisle and Cobb [14].

Data taken with the $200 \mathrm{MeV} / c$ beam, deconvolved using the GEANT model as prior, are shown in figure 5. Different contributions to the systematic uncertainty have been considered: sensitivity to the thickness of the absorber, time of flight cuts used for momentum selection, alignment of the detectors and choice of the fiducial cuts. The time of flight systematics dominate. The scattering widths measured from the scattering distributions projected in the $\mathrm{X}-\mathrm{Z}$ and $\mathrm{Y}-\mathrm{Z}$ planes are $\theta=20.3 \pm 0.2 \mathrm{mrad}$ at $172 \mathrm{MeV} / c, \theta=17.1 \pm 0.2 \mathrm{mrad}$ at $200 \mathrm{MeV} / c$ and $\theta=13.8 \pm 0.1 \mathrm{mrad}$ at $240 \mathrm{MeV} / c$. The preliminary analysis indicates that GEANT4 underestimates the scattering width, while the PDG model overestimates it [15].
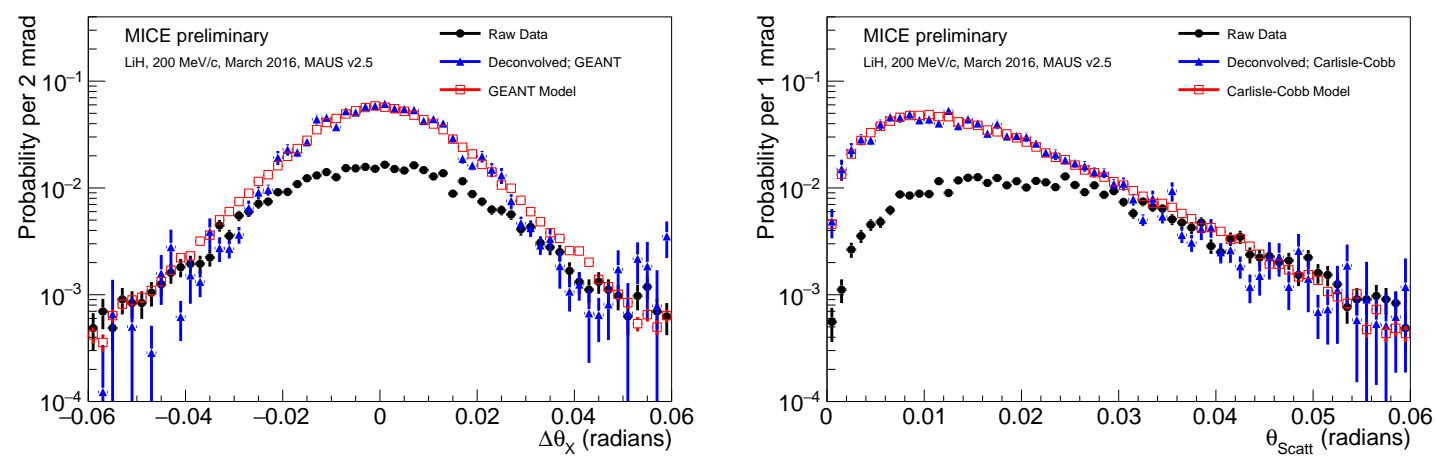

Figure 5: Projected (left) and 3D (right) scattering distributions of $200 \mathrm{MeV} / \mathrm{c}$ muons passing through the LiH absorber.

\section{Study of ionization cooling}

\subsection{Amplitude evolution}

The evolution of phase space density is reported for a single configuration of the cooling apparatus. The transfer line settings were varied to mimic different beam conditions. Results from two transfer line configurations are reported, with the accumulated muon sample having nominal emittances of $3 \mathrm{~mm}$ and $6 \mathrm{~mm}$ at momenta around $140 \mathrm{MeV} / \mathrm{c}$ in the upstream spectrometer solenoid. 
These configurations are denoted ' $3-140$ ' and ' $6-140$ ' respectively. The design optics yields $\beta_{\perp}$ of $0.73 \mathrm{~m}$ at the absorber. Oscillations in $\beta_{\perp}$ occur downstream of the absorber as the downstream matching coils were not powered for this run period, causing some transmission losses.

In order to demonstrate emittance reduction, MICE has measured the distribution of amplitude upstream and downstream of the absorber. The $4 \mathrm{D}$ amplitude of a particle with phase space vector $\mathbf{u}=\left(x, p_{x}, y, p_{y}\right)$ is given by

$$
A_{\perp}=\varepsilon_{\perp}(\mathbf{u}-\boldsymbol{\mu})^{T} \Sigma^{-1}(\mathbf{u}-\boldsymbol{\mu}) .
$$

with $\boldsymbol{\mu}=\left(\langle x\rangle,\left\langle p_{x}\right\rangle,\langle y\rangle,\left\langle p_{y}\right\rangle\right)$, the beam centroid. In order to prevent the tails of the distribution from skewing the core, only those events with amplitude less than $A_{\perp}$ have been included in the calculation of $\boldsymbol{\mu}$ and $\Sigma$ for a given event. While reduction in RMS emittance can be caused by scraping, increase in the number of muons with low amplitude unambiguously demonstrates increase in muon density and cooling.

The amplitude distribution measured upstream and downstream of the absorber is shown in figure 6 for the two different configurations described earlier. For the 3-140 setting, muons with low amplitude upstream of the absorber are observed to move to higher amplitude when measured downstream, as the sample emittance is below equilibrium. Muons sampled from the 6-140 setting are seen to stay with roughly the same amplitude, as the sample is near the equilibrium emittance. There is some dilution due to scraping at high amplitude.
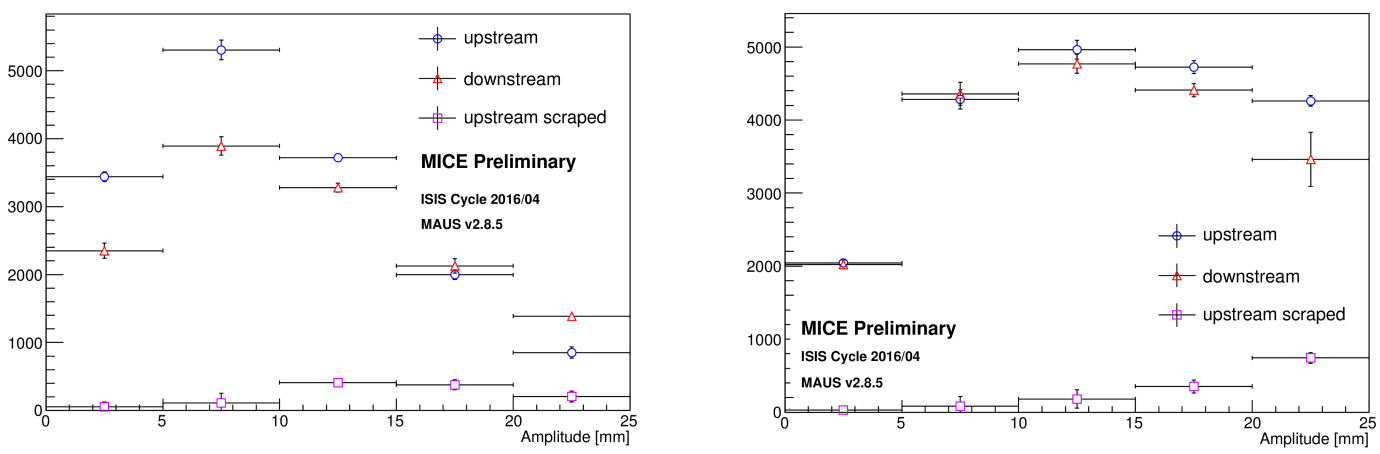

Figure 6: Change in amplitude distribution for the ' $3-140$ ' configuration (left) and for the ' $6-140$ ' configuration (right). The transmission is around $90 \%$ and $80 \%$ respectively. (Blue circles) amplitude of all upstream events; (red triangles) amplitude of all downstream events; (magenta squares) amplitude of upstream events that are not observed downstream.

\subsection{Non-parametric density estimation}

An alternative method to amplitude reconstruction is to use non-parametric techniques to estimate the probability density function of the particle ensemble at the different tracker stations. The $k$ Nearest Neighbours $(k \mathrm{NN})$ estimator identifies the $k$ closest training points to any given test point $\mathbf{x}$, measures the distance, $R_{k}$, to the furthest point and calculates the local density as $\rho(\mathbf{x})=k / \mathscr{V}_{k}$, with $\mathscr{V}_{k}$ the volume of a $d$-ball of radius $R_{k}[17,18]$. Figure 7 shows the 4D phase space density in the $x-p_{x}$ projection, for $\left(y, p_{y}\right)=(0,0)$, at the last downstream tracker station, for a simulated beam of high input emittance and an identical magnetic lattice to the one for which the data was 
presented. The profile exhibits non-Gaussian tails, but the $k \mathrm{NN}$ estimator allows for the reliable identification of the ensemble core.

The evolution of the volume of the $9 \%$ probability contour of this simulated beam is represented on figure 8 . The error band around the central value is purely statistical. The graph exhibits a very clean cooling signal across the absorber that is not biased by transmission losses or nonlinearities in the downstream section. This figure-of-merit can be used to robustly quantify the cooling effect as a function of input emittance and lattice settings.

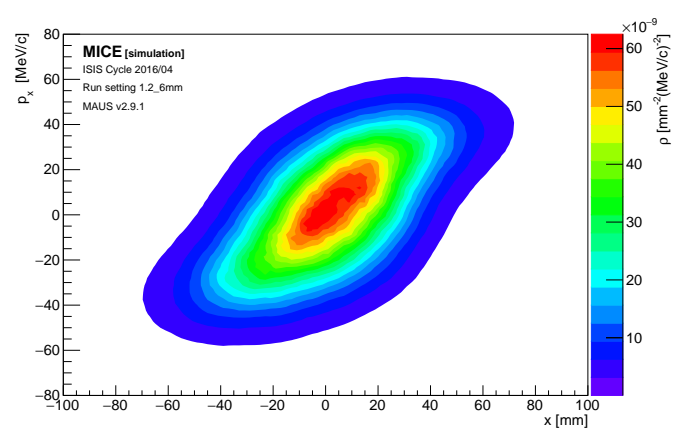

Figure 7: Simulated 4D transverse probability density function in the $x-p_{x}$ projection, for $\left(y, p_{y}\right)=(0,0)$, at the last downstream tracker station.

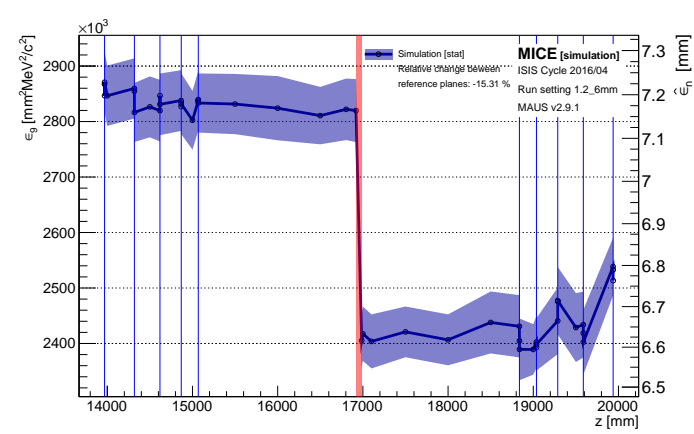

Figure 8: Simulated evolution of the phase space volume of the $9 \%$ probability contour of a large input emittance beam in the MICE cooling channel.

\section{Conclusions}

MICE, at Step IV, will measure the properties of liquid hydrogen and LiH that affect the performance of an ionization-cooling channel. Scattering measurements on $\mathrm{LiH}$ have been performed. The change in amplitude distributions of the MICE muon beam across an absorber has been measured, showing beam dilution below equilibrium emittance and emittance conservation around equilibrium emittance. Non-parametric density estimation techniques offer appealing prospectives to quantitatively assess cooling.

Data taking will continue through 2017 with a liquid hydrogen absorber in a number of different cooling channel configurations. A pair of RF cavities have been constructed. The collaboration seeks to upgrade the existing equipment to demonstrate energy recovery along with emittance reduction.

\section{Acknowledgments}

Work described here has been made possible through generous funding from the Department of Energy and the National Science Foundation (USA), the Istituto Nazionale di Fisica Nucleare (Italy), the Science and Technology Facilities Council (UK), the European Community under the European Commission Framework Programme 7, the Japan Society for the Promotion of Science and the Swiss National Science Foundation. 


\section{References}

[1] IDS-NF Collaboration, M. Apollonio et al., International Design Study for the Neutrino Factory, Nucl.Phys.Proc.Suppl. 229-232 (2012) p.515-515

[2] Neutrino Factory and Muon Collider Collaboration, M. Alsharoa et al., Recent progress in neutrino factory and muon collider research within the Muon collaboration, Phys. Rev. ST Accel. Beams 6 (2003) 081001

[3] V. Parkhomchuck and A. Skrinsky, Cooling Methods for Charged Particle Beams, Rev. Accel. Sci. Tech. 1 no. 1 (2008) p.237

[4] D. Neuffer, Principles and Applications of Muon Cooling, Part. Accel. 14 (1983) p.75-90

[5] D. Stratakis and R. Palmer, Rectilinear six-dimensional ionization cooling channel for a muon collider: A theoretical and numerical study, Phys. Rev. ST Accel. Beams 18 (2015) 031003

[6] MICE Collaboration, MICE: An International Muon Ionization Cooling Experiment, MICE Note 21 (2003)

[7] MICE Collaboration, C. Heidt, The tracker systems for the muon ionization cooling experiment, Nucl. Instr. Meth. 718 (2014), p.560-562

[8] MICE Collaboration, U. Braver et al., MICE: the Muon Ionization Cooling Experiment. Step I: First Measurement of Emittance with Particle Physics Detectors, Proceedings of the DPF-2011 Conference (2011)

[9] MICE Collaboration, D. Adams et al., Pion contamination in the MICE muon beam, JINST 11 (2016) 03001

[10] MICE Collaboration, D. Adams et al., Electron-muon ranger: performance in the MICE muon beam, JINST 10 (2015) 12012

[11] A. Dobbs et al, The Reconstruction Software for the Muon Ionization Cooling Experiment Trackers, J. Phys.: Conf. Ser. 513 (2014) 022008

[12] MuScat Collaboration, W.J. Murray, Comparison of MuScat data with GEANT4, Nucl. Phys. Proc. Suppl. 149 (2005) p.99-103.

[13] GEANT4 Collaboration, S. Agostinelli et al., GEANT4: A Simulation toolkit, Nucl. Instum. Meth. 265 A506 (2003) p.250-303.

[14] T. Carlisle, Step IV of the Muon Ionization Cooling Experiment (MICE) and the multiple scattering of muons, PhD Thesis, Oxford U. (2013)

[15] R. Bayes, Measurements of the Multiple Coulomb Scattering of Muons by MICE, NuFact16 (2016)

[16] S. Peter, Particle penetration and radiation effects, Springer series in Solid State Sciences 151, Berlin-Heidelberg:Springer-Verlag

[17] Y.P. Mack and M. Rosenblatt, Multivariate k-nearest neighbor density estimates, Journal of Multivariate Analysis 9 (1979) p.1-5

[18] D.O. Loftsgaarden and C.P. Quesenberry, A nonparametric estimate of a multivariate density function, The Annals of Mathematical Statistics 363 (1965) p.1049-1051 